\title{
is Research Square \\ Safety and tolerability of prexasertib in Japanese patients with advanced solid tumors
}

Satoru Iwasa

Noboru Yamamoto

Kohei Shitara

Kenji Tamura

Nobuaki Matsubara

Masaomi Tajimi

Aimee B. Lin

Hiroya Asou

Zhihong Cai

Koichi Inoue

Yuko Shibasaki

Kanako Saito

Hiroki Takai

Toshihiko Doi

\section{Video Abstract}

Keywords: prexasertib, cancer, clinical trial, safety, tolerability, adverse events, pharmacokinetics, phase 1, dose-limiting toxicity, neutropenia, anemia, febrile neutropenia, thrombocytopenia, checkpoint kinase 1 inhibitor, CHK1, solid tumors, open-label, stable disease, Japan, Cancer Science

Posted Date: September 20th, 2019

DOI: https://doi.org/10.21203/rs.2.15040/v1

License: (c) (i) This work is licensed under a Creative Commons Attribution 4.0 International License. Read Full License 


\section{Abstract}

A recent report in the journal Cancer Science adds to the growing body of evidence demonstrating that prexasertib is a promising option for several types of cancer. Two prior clinical trials conducted in the United States showed prexasertib has both antitumor activity and an acceptable safety profile. A new clinical trial extends these findings by looking at how the drug performs in a new demographic of patients - namely, Japanese patients with advanced solid tumors. Prexasertib is a novel inhibitor of the protein checkpoint kinase 1 - a serine/threonine kinase that promotes DNA repair, controls initiation of DNA replication, and coordinates mitosis. Blocking the protein's activity prevents cells from resolving replication stress and/or repairing DNA double-strand breaks, which leads to apoptosis. Inhibitors of checkpoint kinase 1 can augment the efficacy of DNA-damaging chemotherapeutics, but they're also being evaluated as single-agent therapies. The phase 1 trial was conducted at the National Cancer Center Hospital and National Cancer Center Hospital East in Japan. Researchers evaluated twelve patients with advanced or metastatic solid tumors who experienced treatment failure with standard therapies. All eligible patients received 80 or $105 \mathrm{mg} / \mathrm{m} 2$ prexasertib on day 1 of a 14- day cycle. The trial investigated the safety, tolerability, pharmacokinetics, and antitumor activity of the drug. The results showed a similar safety and tolerability profile to prior studies. The most common toxicities were hematologic, which were of similar incidence and severity to those observed in non-Japanese patients. Two patients, one in each dose group, experienced dose-limiting toxicities of febrile neutropenia, one grade 4 and the other grade 3 . Both patients recovered and continued the study treatment. The pharmacokinetics of the drug showed dose- and time-independent behavior under both dosage levels tested, consistent with prior reports. In contrast to preceding trials, no patients showed a complete response or partial response. Eight patients had a best overall response of stable disease. Several of the patients had cancer types not previously investigated with prexasertib treatment, including malignant neoplasm of thymus, rhabdomyosarcoma, small intestine carcinoma and transitional cell carcinoma. Overall, the safety findings were consistent with the known safety profile for prexasertib, and tolerability at the recommended dose of $105 \mathrm{mg} / \mathrm{m} 2$ was confirmed in Japanese patients with advanced solid tumors. 